\title{
BMJ Open Is sarcopenia associated with anxiety symptoms and disorders? A systematic review and meta-analysis protocol
}

\author{
Emma C West (D) , ${ }^{1}$ Lana J Williams (D) , ${ }^{1,2}$ Kayla B Corney (D) , ${ }^{1}$ \\ Julie A Pasco (10) 1,2,3,4
}

To cite: West EC, Williams LJ, Corney KB, et al. Is sarcopenia associated with anxiety symptoms and disorders? A systematic review and metaanalysis protocol. BMJ Open 2021;11:e054125. doi:10.1136/ bmjopen-2021-054125

- Prepublication history and additional supplemental material for this paper are available online. To view these files, please visit the journal online (http://dx.doi.org/10.1136/ bmjopen-2021-054125).

Received 07 June 2021 Accepted 13 October 2021

Check for updates

(C) Author(s) (or their employer(s)) 2021. Re-use permitted under CC BY-NC. No commercial re-use. See rights and permissions. Published by BMJ.

${ }^{1}$ IMPACT - Institute for Mental and Physical Health and Clinical Translation, School of Medicine, Deakin University, Geelong, Victoria, Australia

${ }^{2}$ Barwon Health, Geelong, Victoria, Australia

${ }^{3}$ Department of MedicineWestern Health, The University of Melbourne, St Albans, Victoria, Australia

${ }^{4}$ Department of Epidemiology and Preventative Medicine,

Monash University, Melbourne, Victoria, Australia

Correspondence to

Emma C West;

westem@deakin.edu.au

\section{ABSTRACT}

Introduction Sarcopenia is a skeletal muscle disorder characterised by a progressive decline in muscle mass and function (strength and performance). Sarcopenia is associated with numerous adverse health outcomes and has recently been linked to neurological and psychiatric disorders, including dementia and depression. Whether sarcopenia is related to other common psychiatric illnesses, such as anxiety, is unclear. We aim to systematically identify and review the extant literature regarding the association between sarcopenia and anxiety symptomatology and/or disorders (anxiety) in adults. Methods and analysis We will conduct a systematic search across four online databases (CINAHL, Embase, MEDLINE Complete and PsycINF0) from inception to September 2021. Two reviewers will independently confirm study selection and assess methodological quality of included studies. If possible, a meta-analysis will be performed to determine pooled OR for the relationship between sarcopenia and anxiety. If meta-analysis is not possible due to methodological heterogeneity a 'best evidence synthesis' will be performed.

Ethics and dissemination This review will use published data only, thus, ethical approval will not be required.

Findings will be published in a peer-reviewed journal and presented at conferences.

PROSPERO registration number CRD42020209420.

\section{INTRODUCTION}

Sarcopenia is a skeletal muscle disorder characterised by a progressive decline in muscle mass and function (strength and performance). ${ }^{1}$ It is primarily age related and affects approximately $10 \%$ of adults aged $\geq 60$ years. $^{2}$ Sarcopenia is associated with numerous adverse health outcomes including functional decline, ${ }^{3}$ falls,${ }^{3-5}$ fractures, ${ }^{4}$ hospitalisation $^{6}$ and mortality. ${ }^{3} 7$ An emerging body of evidence suggests sarcopenia is also associated with neurological and psychiatric disorders such as cognitive impairment, ${ }^{8} 9$ dementia $^{10}$ and depression. ${ }^{11-13}$

Anxiety disorders are among the most common psychiatric disorders worldwide. ${ }^{14}$ In Australia, reported lifetime prevalence of anxiety disorders is $13.5 \%$ for women ${ }^{15}$

\section{Strengths and limitations of this study}

This study will be the first to systematically assess the literature on the association between sarcopenia and anxiety.

- Two reviewers will independently confirm study selection and assess methodological quality of included studies.

- Meta-analysis is contingent on heterogeneity of available evidence.

- Non-English studies will not be considered, potentially introducing language bias.

and $7.2 \%$ for men. ${ }^{16}$ The economic burden associated with anxiety is substantial to both individuals and society. Total annual healthcare costs associated with anxiety disorders in 2007 was an estimated \$A376 million, which consisted of $\$ \mathrm{~A} 77.9$ million to individuals. ${ }^{17}$ Co-occurrence of physical disorders, including musculoskeletal diseases, is common among individuals with anxiety. ${ }^{18-20}$ However, whether sarcopenia is related to anxiety is unclear.

There are a number of potential mechanisms linking sarcopenia and psychiatric disorders. ${ }^{21}$ Skeletal muscle contractions produce neurotrophic factors, such as brain derived neurotrophic factor, which is known to play a role in mood disorders and has also been implicated in anxiety disorders. ${ }^{22}$ Chronic inflammation is known to play a crucial role in the progression of sarcopenia ${ }^{2324}$ as well as influencing the expression and evolution of anxiety disorders. ${ }^{25}$ Furthermore, sarcopenia and anxiety share a number of common lifestyle risk factors including physical inactivity, poor nutrition and smoking. ${ }^{21}$

Data from recent observational studies investigating associations between sarcopenia and anxiety appear inconsistent. Some studies report no association between muscle function and anxiety, ${ }^{26}$ whereas other studies have reported associations between 
low muscle strength and the onset and persistence of anxiety. ${ }^{28}{ }^{29}$ Evidence regarding other putative components of sarcopenia, such as muscle mass, also appear conflicting. A recent cross-sectional analysis of 113 haemodialysis patients reported an inverse association between loss of muscle mass and high anxiety distress scores. ${ }^{30}$ Conversely, a study of 237 patients with cancer found no association between muscle mass and anxiety scores. ${ }^{31}$ To date, no attempt has been made to synthesise evidence regarding associations between sarcopenia and anxiety. To address this gap in the literature, we will systematically identify and review the extant literature regarding the association between sarcopenia and anxiety in adults.

\section{Objectives}

This systematic review will:

1. Identify published studies that investigate the association between sarcopenia and anxiety.

2. Evaluate the methodological quality of included studies.

3. Collate the evidence and provide a comprehensive synthesis of the findings.

\section{METHODS AND ANALYSIS}

This protocol has been developed following the Preferred Reporting Items for Systematic Reviews and MetaAnalyses (PRISMA) Protocols guidelines. ${ }^{32}$ The methods for this systematic review have been developed according to recommendations from the PRISMA statement. ${ }^{33}$

\section{Inclusion and exclusion criteria}

Studies will be included if they meet the following criteria:

\section{Study design}

Case-control, cross-sectional, cohort designs and clinical trials (baseline data) will be included. Case reports, grey literature and animal studies will be excluded.

\section{Participants}

Studies involving adults aged 18 years or older of any nationality. Community-dwelling, institutionalised and clinical samples will all be eligible for inclusion.

\section{Sarcopenia}

Studies that include a measure of sarcopenia as defined by a professional group, such as: the European Working Group on Sarcopenia in Older Persons ${ }^{134}$; the Asian Working Group for Sarcopenia ${ }^{3536}$; the Foundation for the National Institutes of Health ${ }^{37}$; Special Interest Group ${ }^{38}$; the Society on Sarcopenia, Cachexia and Wasting Disor$\operatorname{ders}^{39}$; International Working Group on Sarcopenia ${ }^{40}$ and Sarcopenia Definition and Outcomes Consortium. ${ }^{41}$ Full definitions proposed by these professional groups are presented in table 1. Given the current absence of a universal definition, studies examining putative components of sarcopenia (muscle mass and muscle function) will also be included.

\section{Anxiety}

Symptoms of anxiety as measured by a validated symptom scale or diagnoses of anxiety-related disorders based on the Diagnostic and Statistical Manual of Mental Disorders or the International Classification of Diseases classification systems. Studies that focus on anxiety related to fear of falling, pain and appearance will be excluded. Studies that assess comorbid depression or general mental health will be excluded.

\section{Search strategy}

We will perform an electronic search across four databases: CINAHL, Embase, MEDLINE Complete and PsycINFO. The search strategy was developed alongside a medical librarian using a combination of free-text terms and controlled vocabulary terms (CINAHL SH/Emtree/ $\mathrm{MeSH} / \mathrm{APA}$ Thesaurus). The search strategy was designed to be broad and inclusive of all conceptualisations of sarcopenia and its putative components. Keywords were drawn from relevant literature and further refined using a 'gold set' of articles. There will be no restrictions on publication date, studies will be included from inception to September 2021. An illustrative example of the search strategy is presented in table 2. Full search strategy adapted for each database is available in online supplemental material. For completeness, we will handsearch the reference lists of included studies and other relevant articles to identify further potentially relevant citations.

\section{Data management and extraction}

We will download the references identified in our electronic searches into EndNote V.X9 reference management software (Thomas Reuters, New York, USA) where duplicates will be manually removed. The remaining references will be exported into Covidence, ${ }^{42}$ an online software used for systematic review data management. Titles and abstracts of records identified in the search will be independently screened for eligibility by two reviewers using a prepiloted screening/selection tool that incorporates the predetermined inclusion criteria described in this protocol. The screening/selection tool will be piloted on approximately 30 titles and abstracts. If there are any conflicts between reviewers' decisions during pilot testing, the review team will meet to discuss and refine the screening/selection tool. Full-text articles will be retrieved for studies that satisfy the eligibility criteria in title and abstract screening. Full-text articles will be independently assessed by two reviewers using the screening/ selection tool with disagreements resolved by discussion until consensus is reached. A third reviewer will be consulted where necessary to reach consensus. A PRISMA flow diagram will be used to document the screening and selection process including reasons for exclusion in line with the predetermined eligibility criteria. ${ }^{33}$

A custom data extraction form will be used to collate information from included studies. The key information to be extracted will include but may not be limited to: (1) study name; (2) country and year; (3) population 
Table 1 Sarcopenia definitions and proposed cut-offs

\begin{tabular}{|c|c|}
\hline Professional group & Sarcopenia definition \\
\hline 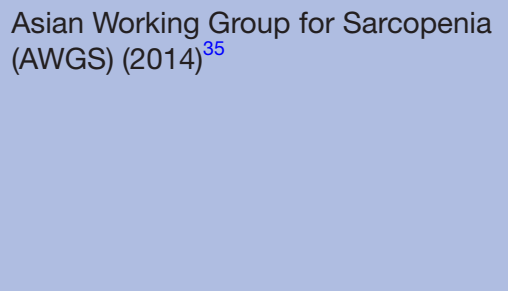 & $\begin{array}{l}\text { Low muscle mass plus low muscle strength and/or low physical performance. } \\
\text { Cut-offs } \\
\text { Handgrip strength }<26 \mathrm{~kg} \text { for males, }<18 \mathrm{~kg} \text { for females } \\
\text { Gait speed }<0.8 \mathrm{~m} / \mathrm{s} \\
\text { Height adjusted muscle mass using dual-energy X-ray absorptiometry }<7.0 \mathrm{~kg} / \mathrm{m}^{2} \text { for } \\
\text { males, }<5.4 \mathrm{~kg} / \mathrm{m}^{2} \text { for females } \\
\text { Height adjusted muscle mass using bioimpedance }<7.0 \mathrm{~kg} / \mathrm{m}^{2} \text { for males, }<5.7 \mathrm{~kg} / \mathrm{m}^{2} \\
\text { for females }\end{array}$ \\
\hline
\end{tabular}

European Working Group on Sarcopenia in Presence of both low muscle mass and low muscle function (strength or performance). Older Persons (EWGSOP) (2010) $\quad$ Cut-offs

- Gait speed $\leq 0.8 \mathrm{~m} / \mathrm{s}$

- Offers examples from literature of various cut-off points for muscle mass and handgrip strength

EWGSOP2 (2019)

Low muscle strength key characteristic, low muscle quantity and quality to confirm diagnosis. Physical performance as indicator of severity.

Cut-offs

- Grip strength $<27 \mathrm{~kg}$ for males, $<16 \mathrm{~kg}$ for females

- Gait speed $\leq 0.8 \mathrm{~m} / \mathrm{s}$

- Short Physical Performance Battery score $\leq 8$

- Timed up and go $\geq 20$ s

- Chair stand $>15 \mathrm{~s}$ for five rises

- 400 m walk test non-completion or $\geq 6$ min for completion

$\begin{array}{ll}\text { Foundation for the National Institutes of } & \text { Low muscle mass and weakness. } \\ \text { Health }(2014)^{37} & \text { Cut-offs } \\ & \text { Appendicular lean mass adjusted for BMI }<0.789 \text { for males, }<0.512 \text { for females } \\ & \text { Gait speed }<0.8 \mathrm{~m} / \mathrm{s} \\ & \text { Grip strength }<26 \mathrm{~kg} \text { for males, }<16 \mathrm{~kg} \text { for females }\end{array}$

International Working Group on Sarcopenia $(2011)^{40}$

Loss of skeletal muscle mass and function.

Cut-offs

- Gait speed less than $1 \mathrm{~m} / \mathrm{s}$

- Appendicular mass relative to height $\leq 7.23 \mathrm{~kg} / \mathrm{m}^{2}$ for males, $\leq 5.67 \mathrm{~kg} / \mathrm{m}^{2}$ for females

$\begin{array}{ll}\text { Sarcopenia Definition and Outcomes } & \text { Muscle weakness defined by low grip strength and slowness defin } \\ \text { Consortium (2020) } & \text { speed. } \\ & \text { Cut-offs } \\ & \text { Maximal grip strength }<35.5 \mathrm{~kg} \text { for males, }<20 \mathrm{~kg} \text { for females } \\ & \text { Grip strength/BMl }<1.05 \text { for males, }<0.79 \text { for females } \\ & \text { Grip strength/total body fat }<1.66 \text { for males, }<0.65 \text { for females } \\ & \text { Grip strength/arm lean mass }<6.1 \text { for males, }<3.26 \text { for females } \\ & \text { Grip strength/body weight }<0.45 \text { for males, }<0.34 \text { for females } \\ & \text { Gait speed }<0.8 \mathrm{~m} / \mathrm{s}\end{array}$

Society on Sarcopenia, Cachexia and Wasting Disorders $(2011)^{39}$

$\begin{array}{ll}\text { Special Interest Group (2010) } & \text { Combined presence of low muscle mass and low muscle function defined by low gait } \\ & \text { speed or well-established functional tests. } \\ & \text { Cut-offs } \\ & \text { Muscle mass percentage of } \geq 2 \mathrm{SD} \text { below the mean measured in young adults of the } \\ & \text { same sex and ethnic background } \\ & <0.8 \mathrm{~m} / \mathrm{s} \text { during } 4 \mathrm{~m} \text { walking test }\end{array}$

Reduced muscle mass, with limited mobility.

Cut-offs

- Lean appendicular mass/height ${ }^{2}$ of $\leq 2$ SD below mean of healthy persons between 20 and 30 years of same ethnic group

- Walking speed $\leq 1 \mathrm{~m} / \mathrm{s}$ or $<400 \mathrm{~m}$ during 6 min walk

Combined presence of low muscle mass and low muscle function defined by low gait Cut-offs

Muscle mass percentage of $\geq 2$ SD below the mean measured in young adults of the

$<0.8 \mathrm{~m} / \mathrm{s}$ during $4 \mathrm{~m}$ walking test

BMI, body mass index. 
Table 2 Illustrative search strategy using Medline complete via EBSCOhost

1. $A B$ anxiety OR TI anxiety OR AB anxious OR TI anxious OR (MM "Anxiety") OR (MH "Anxiety Disorders+")

2. AB sarcopen* OR TI sarcopen* OR AB "muscle mass" OR TI "muscle mass" OR AB "lean mass" OR TI "lean mass" OR AB "skeletal mass" OR TI "skeletal mass" OR (MH "Muscular Atrophy+")

3. AB dynapenia OR TI dynapenia OR AB "muscle weakness" OR TI "muscle weakness" OR AB "muscular weakness" OR $\mathrm{TI}$ "muscular weakness" OR AB "muscle strength" OR TI "muscle strength" OR AB "muscular strength" OR TI "muscular strength" OR AB "grip strength" OR TI "grip strength" OR AB "handgrip strength" OR TI "handgrip strength" OR (MH "Muscle Strength+") OR (MM "Muscle Strength Dynamometer")

4. $\mathrm{AB}$ "gait speed" OR TI "gait speed" OR AB "walking speed" OR TI "walking speed" OR AB "walk test" OR TI "walk test" OR $A B$ "physical performance" OR TI "physical performance" OR AB "timed up and go" OR TI "timed up and go" OR AB "chair stand" OR TI "chair stand" OR AB "limited mobility" OR TI "limited mobility" OR (MM "Walking Speed") OR (MM "Mobility Limitation")

5. 2 OR 3 OR 4

6. 1 AND 5

characteristics; (4) definition and measures of sarcopenia; (5) definition and measures of anxiety; (6) main findings and (7) description of confounders included in the statistical models.

\section{Assessment of methodological quality of included studies}

Two reviewers will independently assess the quality of included studies using the study quality assessment tools published by the US National Heart, Lung and Blood Institute. ${ }^{43}$ Observational study designs will be assessed using the Quality Assessment Tool for Observational Cohort and Cross-Sectional Studies (14 items) and the Quality Assessment of Case-Control Studies (12 items). Clinical trials (baseline data) will be assessed using the Quality Assessment of Controlled Intervention Studies (14 items). We will categorise the overall quality of each study as follows: high $(\geq 70 \%)$, moderate $(40 \%-69 \%)$ or low $(<40 \%)$, with a quality rating of low translating to a high risk of bias. Disagreements between reviewers will be resolved through discussion. If unable to reach agreement, final judgement will be provided by a third reviewer.

\section{Data synthesis}

Where methodological heterogeneity is low, a metaanalysis will be performed to determine pooled OR for the relationship between sarcopenia and anxiety. If sufficient data are available, we will also conduct subgroup analyses. The groups may be designed based on diagnostic criteria for sarcopenia, sex, age and country. The homogeneity of the OR of the included studies will be assessed using $\mathrm{I}^{2}$ statistics. ${ }^{44}$ Sources of heterogeneity will be investigated by removing studies at high risk of bias and comparing different study-level methodological characteristics (eg, sarcopenia definition).

If meta-analysis is not possible due to methodological heterogeneity a 'best evidence synthesis' will be performed instead. This method would determine the combined level of evidence, ranging from 'no evidence' to 'strong evidence', as previously described in the musculoskeletal field. ${ }^{45} 46$

\section{ETHICS AND DISSEMINATION}

This systematic review will use published data only and therefore will not require ethical permission. We aim for completion of the review by June 2022. Findings will be disseminated via publication in a peer-reviewed scientific journal as well as through conference presentations.

Acknowledgements The authors would like to thank Blair Kelly, Medical Librarian at Deakin University, for assisting with development of the search strategy.

Contributors ECW, LJW and JAP conceptualised and edited the research question for this protocol. ECW, LJW, KBC and JAP contributed to development of the methodology, drafting and editing of this manuscript. All authors (ECW, LJW, KBC and JAP) approved the final version for publication.

Funding ECW is supported by a Deakin University Postgraduate Research Scholarship (DUPRS). KBC is supported by the Australian Rotary Health/Bing Taylor PhD Scholarship. LJW is supported by an NHMRC Emerging Leadership Fellowship (1174060).

Competing interests None declared.

Patient consent for publication Not applicable.

Provenance and peer review Not commissioned; externally peer reviewed.

Supplemental material This content has been supplied by the author(s). It has not been vetted by BMJ Publishing Group Limited (BMJ) and may not have been peer-reviewed. Any opinions or recommendations discussed are solely those of the author(s) and are not endorsed by BMJ. BMJ disclaims all liability and responsibility arising from any reliance placed on the content. Where the content includes any translated material, BMJ does not warrant the accuracy and reliability of the translations (including but not limited to local regulations, clinical guidelines, terminology, drug names and drug dosages), and is not responsible for any error and/or omissions arising from translation and adaptation or otherwise.

Open access This is an open access article distributed in accordance with the Creative Commons Attribution Non Commercial (CC BY-NC 4.0) license, which permits others to distribute, remix, adapt, build upon this work non-commercially, and license their derivative works on different terms, provided the original work is properly cited, appropriate credit is given, any changes made indicated, and the use is non-commercial. See: http://creativecommons.org/licenses/by-nc/4.0/.

ORCID iDs

Emma C West http://orcid.org/0000-0001-9559-7003

Lana J Williams http://orcid.org/0000-0002-1377-1272

Kayla B Corney http://orcid.org/0000-0003-4789-0007

Julie A Pasco http://orcid.org/0000-0002-8968-4714 


\section{REFERENCES}

1 Cruz-Jentoft AJ, Bahat G, Bauer J, et al. Sarcopenia: revised European consensus on definition and diagnosis. Age Ageing 2019;48:16-31.

2 Shafiee G, Keshtkar A, Soltani A, et al. Prevalence of sarcopenia in the world: a systematic review and meta- analysis of general population studies. J Diabetes Metab Disord 2017;16:21.

3 Beaudart C, Zaaria M, Pasleau F, et al. Health outcomes of sarcopenia: a systematic review and meta-analysis. PLoS One 2017;12:e0169548.

4 Yeung SSY, Reijnierse EM, Pham VK, et al. Sarcopenia and its association with falls and fractures in older adults: a systematic review and meta-analysis. J Cachexia Sarcopenia Muscle 2019;10:485-500.

5 Pasco JA, Brennan Olsen SL, et al. Low lean tissue mass and physical performance as markers of sarcopenia in older men and women. J Gerontol Geriatr Res 2016;5:306.

6 Zhang X, Zhang W, Wang C, et al. Sarcopenia as a predictor of hospitalization among older people: a systematic review and metaanalysis. BMC Geriatr 2018;18.

7 Pasco JA, Mohebbi M, Holloway KL, et al. Musculoskeletal decline and mortality: prospective data from the Geelong osteoporosis study. J Cachexia Sarcopenia Muscle 2017;8:482-9.

8 Chang K-V, Hsu T-H, Wu W-T, et al. Association between sarcopenia and cognitive impairment: a systematic review and meta-analysis. $J$ Am Med Dir Assoc 2016;17:1164

9 Sui SX, Holloway-Kew KL, Hyde NK. Muscle strength and gait speed rather than lean mass are better indicators for poor cognitive function in older men. J Cachexia Sarcopenia Muscle 2020;11:690-7.

10 Pacifico J, Geerlings MAJ, Reijnierse EM, et al. Prevalence of sarcopenia as a comorbid disease: a systematic review and metaanalysis. Exp Gerontol 2020;131:110801.

11 Chang K-V, Hsu T-H, Wu W-T, et al. Is sarcopenia associated with depression? A systematic review and meta-analysis of observational studies. Age Ageing 2017;46:738-46.

12 Marques A, Gomez-Baya D, Peralta M, et al. The effect of muscular strength on depression symptoms in adults: a systematic review and meta-analysis. Int J Environ Res Public Health 2020;17:5674.

13 Pasco JA, Sui SX, West EC. Operational definitions of sarcopenia should consider depressive symptoms. JCSM Clin Rep 2021;6:62-8.

14 Stein DJ, Scott KM, de Jonge P, et al. Epidemiology of anxiety disorders: from surveys to nosology and back. Dialogues Clin Neurosci 2017;19:127-36.

15 Williams L, Jacka F, Pasco J, et al. The prevalence of mood and anxiety disorders in Australian women. Australas Psychiatry 2010;18:250-5

16 Williams LJ, Jacka FN, Pasco JA, et al. The prevalence and age of onset of psychiatric disorders in Australian men. Aust N Z J Psychiatry 2016;50:678-84.

17 Lee Y-C, Chatterton ML, Magnus A, et al. Cost of high prevalence mental disorders: findings from the 2007 Australian national survey of mental health and wellbeing. Aust N Z J Psychiatry 2017;51:1198-211.

18 Teesson M, Mitchell PB, Deady M, et al. Affective and anxiety disorders and their relationship with chronic physical conditions in Australia: findings of the 2007 national survey of mental health and wellbeing. Aust N Z J Psychiatry 2011;45:939-46.

19 Heikkinen J, Honkanen R, Williams L, et al. Depressive disorders, anxiety disorders and subjective mental health in common musculoskeletal diseases: a review. Maturitas 2019:127:18-25.

20 Williams LJ, Bjerkeset O, Langhammer A, et al. The association between depressive and anxiety symptoms and bone mineral density in the general population: the HUNT study. J Affect Disord 2011;131:164-71.

21 Pasco JA, Williams LJ, Jacka FN, et al. Sarcopenia and the common mental disorders: a potential regulatory role of skeletal muscle on brain function? Curr Osteoporos Rep 2015;13:351-7.

22 Castrén E, Kojima M. Brain-derived neurotrophic factor in mood disorders and antidepressant treatments. Neurobiol Dis 2017;97:119-26.

23 Dalle S, Rossmeislova L, Koppo K. The role of inflammation in agerelated sarcopenia. Front Physiol 2017;8:1045.

24 Pasco J. Age-related changes in muscle and bone. In: Duque G, ed. Osteosarcopenia: bone, muscle and fat interactions. Springer Nature Switzerland AG, 2019: 45-71.
25 Moylan S, Eyre HA, Maes M, et al. Exercising the worry away: how inflammation, oxidative and nitrogen stress mediates the beneficial effect of physical activity on anxiety disorder symptoms and behaviours. Neurosci Biobehav Rev 2013;37:573-84.

26 Staples WH, Kays A, Richman R. Examination of the correlation between physical and psychological measures in communitydwelling older adults. Clin Interv Aging 2020;15:293-300.

27 Machado FVC, Schneider LP, Fonseca J, et al. Clinical impact of body composition phenotypes in patients with COPD: a retrospective analysis. Eur J Clin Nutr 2019;73:1512-9.

28 Gordon BR, McDowell CP, Lyons M, et al. Associations between grip strength and generalized anxiety disorder in older adults: results from the Irish longitudinal study on ageing. J Affect Disord 2019;255:136-41.

29 Carvalho AF, Maes M, Solmi M, et al. Is dynapenia associated with the onset and persistence of depressive and anxiety symptoms among older adults? Findings from the Irish longitudinal study on ageing. Aging Ment Health 2021;25:468-75.

30 Alston H, Burns A, Davenport A. Loss of appendicular muscle mass in haemodialysis patients is associated with increased self-reported depression, anxiety and lower general health scores. Nephrology 2018;23:546-51.

31 Nipp RD, Fuchs G, El-Jawahri A, et al. Sarcopenia is associated with quality of life and depression in patients with advanced cancer. Oncologist 2018;23:97-104.

32 Moher D, Shamseer L, Clarke M, et al. Preferred reporting items for systematic review and meta-analysis protocols (PRISMA-P) 2015 statement. Syst Rev 2015;4:1.

33 Page MJ, McKenzie JE, Bossuyt PM, et al. The PRISMA 2020 statement: an updated guideline for reporting systematic reviews. BMJ 2021;372:n71.

34 Cruz-Jentoft AJ, Baeyens JP, Bauer JM, et al. Sarcopenia: European consensus on definition and diagnosis: report of the European Working group on sarcopenia in older people. Age Ageing 2010;39:412-23.

35 Chen L-K, Liu L-K, Woo J, et al. Sarcopenia in Asia: consensus report of the Asian working group for sarcopenia. J Am Med Dir Assoc 2014;15:95-101.

36 Chen L-K, Woo J, Assantachai P, et al. Asian working group for sarcopenia: 2019 consensus update on sarcopenia diagnosis and treatment. J Am Med Dir Assoc 2020;21:300-7.

37 Studenski SA, Peters KW, Alley DE, et al. The FNIH sarcopenia project: rationale, study description, conference recommendations, and final estimates. J Gerontol A Biol Sci Med Sci 2014;69:547-58.

38 Muscaritoli M, Anker SD, Argilés J, et al. Consensus definition of sarcopenia, cachexia and pre-cachexia: joint document elaborated by Special Interest Groups (SIG) "cachexia-anorexia in chronic wasting diseases" and "nutrition in geriatrics". Clin Nutr 2010;29:154-9.

39 Morley JE, Abbatecola AM, Argiles JM, et al. Sarcopenia with limited mobility: an international consensus. J Am Med Dir Assoc 2011;12:403-9.

40 Fielding RA, Vellas B, Evans WJ, et al. Sarcopenia: an undiagnosed condition in older adults. current consensus definition: prevalence, etiology, and consequences. International Working group on sarcopenia. J Am Med Dir Assoc 2011;12:249-56.

41 Bhasin S, Travison TG, Manini TM, et al. Sarcopenia definition: the position statements of the sarcopenia definition and outcomes Consortium. J Am Geriatr Soc 2020;68:1410-8.

42 Covidence systematic review software. Veritas health innovation. Melbourne, Australia. www.covidence.org

43 National Institutes of Health. National heart, lung and blood Institute quality assessment tool for observational cohort and cross-sectional studies. Available: https://www.nhlbi.nih.gov/ health-topics/study-quality-assessment-tools [Accessed 11 May 2021].

44 Higgins JPT, Thompson SG, Deeks JJ, et al. Measuring inconsistency in meta-analyses. BMJ 2003;327:557-60.

45 Brennan SL, Pasco JA, Urquhart DM, et al. The association between socioeconomic status and osteoporotic fracture in population-based adults: a systematic review. Osteoporos Int 2009;20:1487-97.

46 Brennan SL, Pasco JA, Urquhart DM, et al. The association between urban or rural locality and hip fracture in community-based adults: a systematic review. J Epidemiol Community Health 2010;64:656-65. 